ФГБНУ Научно-исследовательский институт ревматологии им. В.А. Насоновой, Москва, Россия

Проведен одномоментный скрининг случайных выборок мужчин в возрасте 50 лет и старше, проживавших в пяти городах (Архангельск, Мурманск, Тюмень, Красноярск, Челябинск) 3-х федеральных округов РФ, с целью оценки частоты встречаемости отдельных факторов риска (ФР) остеопоротических переломов. В результате обследования 1330 мужчин установлено, что наиболее распространенными были следующие факторы: курение, низкоэнергетические переломы в анамнезе, вторичные причины ОП, недостаточное потребление кальция с продуктами питания. Мужчины в СФО и СЗФО курили чаще, чем лица из УФО ( $\mathrm{p}=0,0011$ и р=0,047, соответственно), распространенность других ФР по регионам не различалась. Остеопороз по данным периферической рентгеновской денситометрии выявлялся чаще у жителей УФО (26\%), чем у мужчин

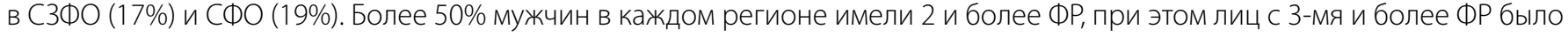
достоверно больше в УФО, а в возрасте 50-59 лет - в С ФО. 85 \% мужчин в этих регионах не получали с продуктами питания адекватного количества кальция.

кЛЮчЕВЫЕ СлОВА: Остеопороз; факторы риска переломов; потребление кальция с пищей; периферическая рентгеновская денситометрия;

\title{
OSTEOPOROSIS AND OSTEOPOROTIC FRACTURES RISK FACTORS IN MEN AGED 50 YEARS AND OLDER
}

\author{
(c) O.A. Nikitinskaya, N.V. Toroptsova
}

Research Institute of Rheumatology named after V.A. Nasonova, Moscow, Russia

Cross-sectional screening of random samples of men aged 50 years and older living in five cities (Arkhangelsk, Murmansk, Tyumen, Krasnoyarsk, and Chelyabinsk) from three Federal Districts (FD) of the Russian Federation was conducted to evaluate the frequency of individual risk factors (RF) of osteoporotic fractures among them. Total 1330 men were included in the study. We found that the most common were the following RF: smoking, prior fragility fractures after age 40, secondary causes of OP, insufficient calcium intake. Significantly more smokers were among males in Siberian (S) FD and North -Western (NW)FD compared with those from Ural $(U) F D(p=0,0011$ and $p=0,047$, respectively), the frequency of other RF did not differ depending on region. The pprevalence of osteoporosis according to peripheral x-ray densitometry of the proximal forearm was higher among residents of the UFD (26\%) than among men in the NWFD (17\%) and SFO (19\%). More than 50\% of men in each region had 2 or more RF, while those with 3 or more RF were significantly more in the UFD at any age, and at the age of 50-59 years - in the NWFD. Calcium intake was inadequate in $85 \%$ of the respondents in these regions.

KEYWORDS: Osteoporosis; risk factors of osteoporotic fractures; calcium intake; peripheral x-ray densitometry;

\section{ОБОСНОВАНИЕ}

Проблема остеопороза (ОП) у мужчин в последние годы становится все более актуальной и социально значимой. Несмотря на то, что ОП у мужчин встречается реже, чем среди женщин, уровень смертности, обусловленный этим заболеванием, у них выше: например, в течение первого года после перелома бедра летальность составляла 37,5\%, что на 51\% было больше по сравнению с таковым показателем у женщин [1].

По данным Международного фонда остеопороза в странах Европейского союза в 2010 г в соответствии с критериями ВОЗ ОП имели 5,5 млн. мужчин. В целом частота ОП среди европейских мужчин в возрасте 50 лет и старше составляла 6,0\%, она увеличивалась с возрастом и среди лиц в возрасте старше 80 лет достигала уже 16,6\% [2]. В представленном Hernlund E и соавт. отчете по ОП в Европейских странах на долю мужчин приходилось 34\% всех ОП-переломов [2]. В другой работе по оценке распростра- нённости ОП и его осложнений в мире этот показатель был еще выше - 39\% [3].

Основополагающее эпидемиологическое исследование, проведенное в Институте ревматологии в конце XX столетия показало, что у мужчин в возрасте 50 лет и старше ОП по данным двухэнергетической рентгеновской абсорбциометрии (DXA) двух областей скелета выявлялся у 26,9\% обследованных лиц, при этом его распространенность при определении минеральной плотности кости (МПК) шейки бедра составляла 17,3\%, а по результатам измерения МПК поясничного отдела позвоночника - 11,5\%. Частота переломов костей периферического скелета при низком уровне травмы составляла 11,6\%, а распространенность переломов позвонков - 10,3\%, при этом у них не было выявлено зависимости частоты переломов позвонков от возраста [4]. В другой эпидемиологической работе, выполненной в Ярославле, анализ частоты переломов проксимального отдела бедра среди лиц моложе 65 лет продемонстрировал, что чаще они происходили у мужчин, чем у женщин [5]. 
На основании эпидемиологических данных, полученных в мире, было рассчитано, что в возрасте 50 лет и старше у одного из четырех мужчин в течение последующей жизни может произойти хотя бы один перелом, связанный с ОП, и вероятность такого перелома выше, чем риск развития рака предстательной железы [6].

ОП у мужчин может быть первичным, связанным с возрастом и возрастной потерей МПК вследствие дефицита эстрогенов и андрогенов, и вторичным, обусловленным большим количеством разных причин, ведущими из которых являются злоупотребление алкоголем, длительное применение глюкокортикоидов (ГК), гипогонадизм. Показано, что на вторичный ОП приходится 40\% всех случаев данного заболевания у мужчин [7].

У мужчин, как и у женщин, диагностика ОП, основанная на определении низкой МПК, не позволяет в полной мере выявить лиц с высоким риском перелома. Так, в одном из исследований было показано, что значения МПК менее -2,5 СО имели только 21\% пожилых мужчин, ранее перенесших низкоэнергетические периферические переломы, и 39\% - с уже случившимся переломом шейки бедренной кости [8]. В другой работе выявили, что только 24\% мужчин с переломами позвонков имели по данным денситометрии ОП, в то время как среди лиц с низкой МПК, соответствующей критериям ОП, переломы позвонков были обнаружены лишь у 1,5\% больных [9]. Поэтому не случайно, что в последнее десятилетие исследования у мужчин были направлены на выявление факторов риска (ФР), ассоциированных не только с низкой МПК, но и с переломами. По данным 4-х летнего проспективного наблюдения 5995 мужчин в возрасте 65 лет и старше (MrOS) увеличение риска периферических переломов независимо от значений МПК общего показателя бедренной кости было связано с такими факторами, как предшествующие переломы в возрасте после 50 лет, возраст 80 лет и старше, прием трициклических антидепрессантов, наличие депрессии, падения в течение предшествующего года и снижением мышечной функции. Наличие 3-х и более из перечисленных ФР повышало риск периферических переломов у мужчин в 5 раз, а их сочетание с низкой МПК - в 15 раз [10].

Опубликованный в 2012г. систематический обзор с мета-анализом ФР ОП-переломов показал, что увеличение возраста, низкий индекс массы тела (ИМТ), злоупотребление алкоголем, курение, длительный прием ГК, предшествующие переломы, падения в течение последнего года, гипогонадизм, инсульт и диабет у мужчин оказывали значимое влияние на повышение риска переломов. При этом такие факторы, как предшествующие переломы, падения, гипогонадизм и перенесенный инсульт, увеличивали этот риск больше, чем возраст, ИМТ, вредные привычки и прием ГК [11].

ОП представляет собой мультифакториальное заболевание и, как показали исследования, факторы, влияющие на риск перелома у мужчин отчасти схожи с теми, которые были определены как значимые у женщин. Поэтому Международным фондом ОП для выявления среди мужчин лиц с высоким риском перелома - кандидатов на антиостеопоротическую терапию, было предложено использовать алгоритм расчета 10-летнего абсолютного риска перелома - FRAX ${ }^{\oplus}$. Эта программа начала внедряться в российскую клиническую практику с 2012г., однако крупномасштабных исследований по оценке распространенности ФР
ОП-переломов, включенных в данный алгоритм, у мужчин, проживающих в различных регионах нашей страны, до последнего времени не было. Проведенный поиск выявил лишь одну эпидемиологическую работу, выполненную в 8-ми городах пяти регионов РФ по единому дизайну, в которой были представлены данные о частоте ФР у мужчин в возрасте 40-69 лет, что было отмечено как ограничение данного исследования [12].

\section{ЦЕЛЬ}

Установить частоту встречаемости отдельных ФР ОП и их распространенность по данным рентгеновской денситометрии дистального отдела предплечья у мужчин в возрасте 50 лет и старше в трех регионах страны.

\section{МЕТОДЫ}

В рамках проведения социальной программы «Остеоскрининг Россия» на базе районных поликлиник были созданы случайные кластерные пропорционально стратифицированные выборки жителей в возрасте 40 лет и старше различных городов РФ с численностью населения более 100 000. В дальнейшем анализировались выборки городов, в которых отвечаемость населения на приглашение к исследованию составила не менее $80 \%$.

В данной статье представлены данные, полученные на выборках мужчин из в 3-х регионов РФ: Северо-Западный федеральный округ (СЗФО) (Архангельск, Мурманск), Уральский федеральный округ (УФО) (Тюмень, Челябинск) и Сибирский федеральный округ (СФО) (Красноярск). Включены 1330 мужчин в возрасте 50 лет и старше (средний возраст составил 61,8土8,5 лет). Все лица подписали информированное согласие на участие в исследовании.

Обследование проводилось с использованием унифицированной анкеты, включавшей факторы риска ОП-переломов, входящих в алгоритм FRAX, и дополнительные вопросы по питанию для оценки суточного потребления кальция с пищей. Респонденты указывали возраст, вес, рост, наличие следующих факторов: предшествующих переломов при минимальной травме (падение с высоты собственного роста) или спонтанные в возрасте после 40 лет, переломов бедра у родителей, курения на момент опроса, приема ГК более 3 месяцев в дозе преднизолона 5 мг и выше в любой период жизни или в настоящее время, ревматоидного артрита (РА), болезни Крона, неспецифического язвенного колита, сахарного диабета I типа, гиперпаратиреоза, гипертиреоза, хронического гепатита или цирроза печени, злоупотребления алкоголем (ежедневный прием более 450 мл пива или 360 мл вина или 90 мл крепких напитков). Опросники заполнялись участниками самостоятельно во время визита в поликлинику с последующей проверкой правильности заполнения предварительно обученной медицинской сестрой. Кроме анкетирования мужчинам было проведено денситометрическое исследование МПК дистального отдела предплечья с помощью периферического рентгеновского остеоденситометра Osteometer Meditech DTX-200.

Статистический анализ выполнен с помощью пакета программ Statistica 10.0 StatSoft для Windows с использованием параметрических и непараметрических методов сравнения. Статистически значимыми считали различия при $\mathrm{p}<0,05$. 
Таблица 1. Частота факторов риска ОП и переломов среди мужчин 50 лет и старше 3-х регионов России

\begin{tabular}{|c|c|c|c|c|}
\hline Фактор риска & 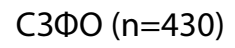 & СФО $(n=432)$ & УФО $(\mathrm{n}=468)$ & $p$ \\
\hline Ср.возраст (лет) & $61,9 \pm 7,3$ & $61,6 \pm 8,7$ & $62,2 \pm 8,5$ & НД \\
\hline ИМТ <20 кг/м² (n\%) & $10(2,5)$ & $18(4,2)$ & $18(3,8)$ & НД \\
\hline Переломы (n\%) & $112(26)$ & 130(30) & $118(25)$ & НД \\
\hline Переломы бедра у родителей (n\%) & $8(1,9)$ & $6(1,4)$ & $6(1,4)$ & НД \\
\hline Курение (n\%) & 138(32) & 156(36) & 122(26) & $<0,05$ \\
\hline Прием глюкокортикоидов & $22(5,1)$ & $26(6,0)$ & $28(5,9)$ & НД \\
\hline Ревматоидный артрит (n\%) & $6(1,4)$ & $6(1,4)$ & $6(1,4)$ & НД \\
\hline Другие вторичные причины (n\%) & $72(17)$ & $82(19)$ & $70(15)$ & НД \\
\hline Избыточное потребление алкоголя (n\%) & $22(5,1)$ & $20(4,6)$ & $23(4,9)$ & НД \\
\hline
\end{tabular}

\section{РЕЗУЛЬТАТЫ}

Среди обследованных мужчин 545 (41\%) лиц находились в возрасте 50-59лет, 452 (34\%) - в возрасте 60-69 лет, 293(22\%) - в возрасте 70-79 лет, 40 (3\%) - в возрасте 80 лет и старше.

В целом во всей выборке наиболее распространенными клиническими ФР оказались курение (31,3\%), переломы в анамнезе (27\%), любые причины вторичного ОП - сахарный диабет I и || типа, гиперпаратиреоз, гипертиреоз, хронический гепатит и цирроз печени (16,8\%). Среди опрошенных мужчин 5,7\% получали ГК, 4,9\% - злоупотребляли алкоголем, 3,5\% имели ИМТ ниже 20 кг/м2, на переломы бедра у родителей указали 1,5\% анкетированных лиц, а 1,4\% респондентов страдали ревматоидным артритом (РА).

При сравнении полученных данных по регионам (таблица) не было обнаружено достоверных различий по распространенности большинства ФР кроме частоты курения. Так, оказалось, что мужчины в СФО и СЗФО курили чаще, чем проживавшие в УФО ( $p=0,0011$ и $p=0,047$, соответственно).

Более 50\% мужчин в каждом регионе имели 2 и более ФР ОП-переломов, из них лиц с 3-мя и более ФР было 27,4\%, при этом последних было достоверно больше в УФО по сравнению с таковыми в С $Ф О(p=0,002)$ (рис.1). В УФО количество лиц, имевших ФР остеопороза и ОП-переломов, увеличивалось с возрастом ( $<<0,05)$, аналогичная тенденция наблюдалась и в СФО (рис.2). В СЗФО не было выявлено четкой связи накопления числа ФР у более пожилых лиц: мужчин, имевших 3 и более ФР ОП-переломов, в возрасте 50-59 лет было больше, чем в возрастных группах 60-69лет и 70-79 лет. При этом установлено, что в воз-

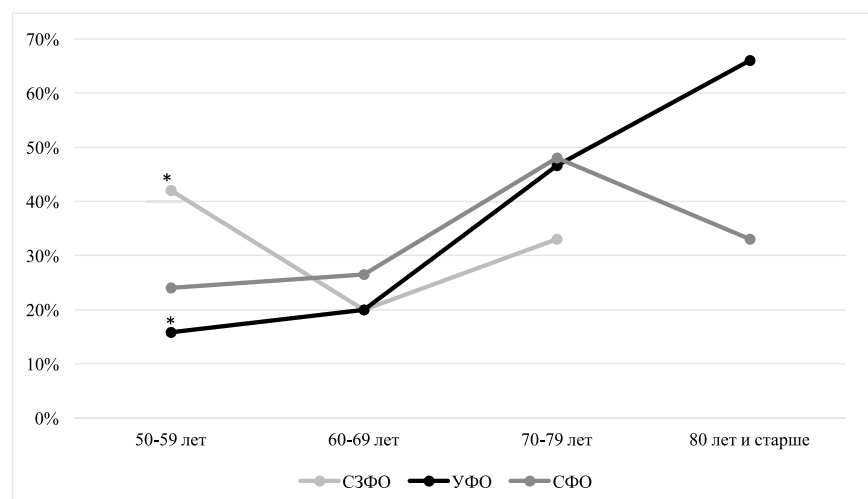

Рис. 1. Встречаемость факторов риска остеопоротических переломов у мужчин, проживающих в СЗФО, УФО и СФО: *p=0,002 для 3-х и более факторов риска у мужчин в СЗФО по сравнению с УФО. расте 50-59 лет в СЗФО мужчины достоверно чаще имели большее количество ФР, чем представители из УФО.

В возрасте 50-59 лет во всех ФО курящие мужчины выявлялись чаще: 78 \% лиц от всех куривших в С ЗФО, 54\% в УФО и 48\% - в СФО. Кроме этого, в этом возрасте переломы в анамнезе имел примерно каждый третий мужчина

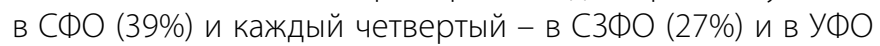
(25\%). Даже несмотря на выявленный небольшой процент лиц, злоупотреблявших алкоголем, среди анкетированных нами представителей мужского пола, надо отметить, что большинство из них были именно в возрасте 50-59 лет.

Потребление кальция во всех изучаемых регионах было низким, только 15 \% мужчин получали с продуктами питания 1000 мг кальция и более. Однако, средние показатели потребления этого макроэлемента с пищей среди мужчин СЗФО (752士314 мг) были выше, чем среди анкетированных лиц из двух других регионов ( $<<0,0001)$,

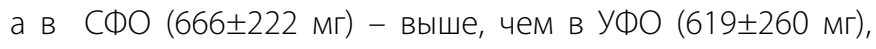
где оно оказалось самым низким ( $p=0,0113)$. Во всех ФО отмечена тенденция к увеличению потребления кальция, преимущественно за счет молочных продуктов, с возрастом: в 50-59 лет мужчины получали кальция меньше, чем в других возрастных группах, а в 60-69 лет - меньше, чем 70-79 лет, но без статистически значимых разли-

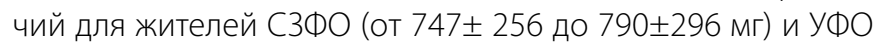

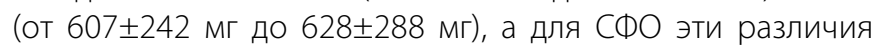

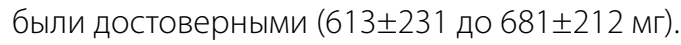

Денситометрическое обследование показало, что ОП дистального отдела предплечья выявлен у 21\% обследованных мужчин, при этом в УФО частота ОП составила 26\%, что было достоверно больше, чем среди жителей СФО и С ЗФО, где Он встречался у $19 \%$ и 17\%, соОтветственно $(p=0,002)$. Кроме этого, отмечена слабая отрицательная

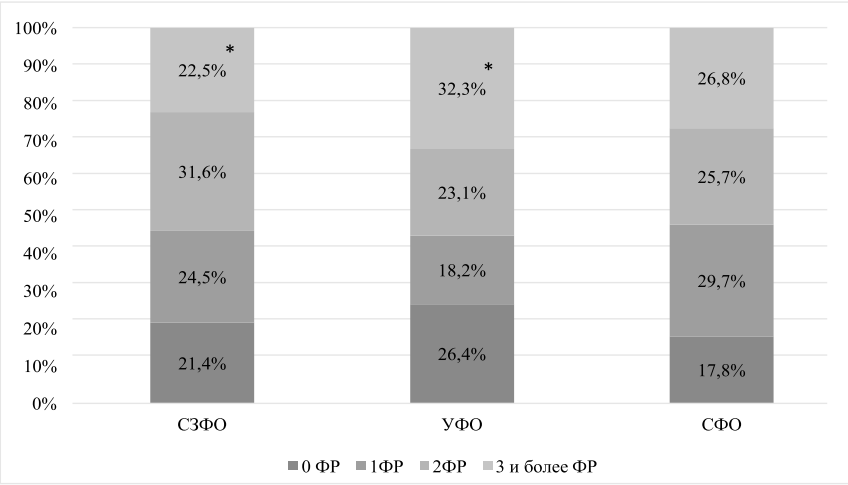

Рис. 2. Встречаемость трёх и более факторов риска остеопоротических переломов в зависимости от возраста: *p=0,007 для СЗФО и УФО. 
линейная корреляция между количеством ФР и показателями МПК ( $r=-0,3, p<0,05)$. У мужчин с 2-мя и более ФР шанс обнаружения низкой МПК, соответствующей уровню ОП, был в 2,7 раза выше (ОШ= 2,71 [1,1;6,7], p=0,026) по сравнению с теми, кто не имел ФР ОП-переломов.

\section{ОБСУЖДЕНИЕ}

Наше исследование показало, что курение у мужчин было самым распространенным ФР и встречалось у 31,3\% анкетированных лиц. Данные, полученные Скрипниковой И.А. и соавт. В другой российской эпидемиологической работе ЭССЕ-РФ, в рамках которой изучались ФР ОП-переломов у мужчин, также был показан высокий процент курящих среди опрошенных людей (34,8\%) [12]. В то же самое время, распространенность таких ФР, как предшествующие переломы, причины вторичного ОП, прием ГК, низкий ИМТ, среди мужчин, участвовавших в нашем анкетировании была в 1,5-2 раза выше по сравнению с результатами, полученными в исследовании ЭССЕ-РФ. Только частота выявления больных РА и лиц, злоупотреблявших алкоголем, по данным обоих опросов практически не различались (1,4\%/1,9\% и 4,9\%/5,2\%, соответственно). Разница в распространенности ряда ФР могла быть обусловлена возрастом лиц, принимавших участие в обоих исследованиях, т.к. известно, что частота большинства ФР ассоциирована с увеличением возраста. Так, в ЭССЕ-РФ были представлены результаты, полученные при анкетировании более молодых мужчин (40-69 лет), средний возраст которых составил 53года, в то время как опрос в нашем исследовании проводился среди мужчин более старших возрастных групп и средний возраст участников был 61год.

Так как обе работы проводились в одних и тех же регионах, хотя в СЗФО и УФО в разных городах, у нас была возможность сравнить полученные результаты по распространенности изученных ФР ОП-переломов. Как и на общей выборке, так и по регионам по данным двух исследований получено совпадение по частоте курения среди мужчин. В С ЗФО, который был представлен Вологдой в ЭССЕ-РФ и Архангельском и Мурманском в нашем исследовании «Остеоскрининг Россия» (ОС-Р), курили 36,3\% и 32\% мужчин, соответственно. В УФО курение отмечено у 27,4\% лиц, проживавших в Тюмени (ЭССЕ-РФ), и у 26\% участников обследования из 2-х городов в ОС-Р. А в Красноярске (СФО), где проводилось оба исследования, но на разных выборках мужчин, по данным ЭССЕ-РФ курили 34,7\% мужчин, а по нашим данным - 36\% респондентов.

По другим ФР данные обоих исследований различались. Например, по СЗФО только частота переломов в анамнезе и процент лиц с РА были схожи: 25,2\% и 1,4\% в Вологде (ЭССЕ-РФ), соответственно, 26\% и 1,4\% - в Архангельске и Мурманске (ОС-Р). В УФО лишь по одному ФР были получены близкие по значению результаты и им оказались переломы бедра у родителей, которые были отмечены в 1,6\% случаев в ЭССЕ-РФ и 1,4\% - в ОС-Р. В Красноярске в результате двух анкетирований установлено, что только ИМТ менее 20 кг/м² определялся с одинаковой частотой 4,1\% (ЭССЕ-РФ) и 4,2\% (ОС-Р), а распространенность других факторов существенно варьировала.

В исследовании ЭССЕ-РФ переломы в анамнезе среди мужчин, жителей Тюмени (УФО) и Красноярска (СФО), выявлялись лишь у 7,1 и 7,9\% лиц, однако в нашей рабо- те 25 и 30\% анкетированных мужчин этих же ФО отметили, что они ранее перенесли перелом при минимальной травме. В недавнем ретроспективном исследовании по амбулаторным картам пациентов мужского пола старше 40 лет, проведенном в Красноярске, было установлено, что 34\% из них имели переломы, половина из которых приходилась на переломы позвонков и 10\% - на перелом шейки бедренной кости [13]. Как видно, данные этой работы согласуются с результатами, полученными в ходе проведенного нами OC-Р. Еще ряд исследований, выполненных в нашей стране в последние годы, подтверждают, что переломы в анамнез у мужчин 50 лет и старше явление не редкое. Так, было показано, что 38,24\% мужчин с переломом бедра имели ранее переломы позвонков [14], а 42\% мужчин, наблюдавшихся с диагнозом сахарный диабет II типа, указывали на наличие переломов различной локализации в анамнезе [15].

Нами обнаружено, что такие ФР, как курение, злоупотребление алкоголем и недостаточное потребление кальция с пищей чаще выявлялись среди лиц мужского пола в возрасте 50-59 лет. Аналогичные данные были получены при изучении факторов, влияющих на заболеваемость переломами проксимального отдела бедренной кости у мужчин, в Ярославле [14]. Оказалось, что высокая заболеваемость этими переломами в возрасте до 70 лет была связана у них с наличием большего количество ФР, включая злоупотребление алкоголем, курение и недостаточное потребление кальция с продуктами питания. В ЭССЕ-РФ также было отмечено, что избыточное потребление алкоголя и курение определялись чаще в более молодых возрастных группах мужчин, чем у лиц старше 60 лет [12].

Частота ОП у мужчин в нашем исследовании составила 21\%, но следует обратить внимание, что мы использовали во всех регионах в качестве скринирующего метода определения МПК периферическую рентгеновскую денситометрию. Наши данные оказались чуть выше, чем в ранее проведенной работе, оценивавшей МПК в аксиальных отделах скелета, где он определялся в 11,5\% и 17,3\% в поясничном отделе позвоночника и шейке бедренной кости, соответственно [4]. Наиболее высокий показатель частоты ОП был в УФО - 26\%, в этом регионе ОП встречался достоверно чаще, чем среди мужского населения других изучаемых в ОС-Р регионов.

По данным Российского мониторинга экономического положения и здоровья населения в 2005г. самый высокий уровень потребления кальция (75,5\% от физиологической нормы) был отмечен у мужчин в возрасте 45-55 лет [16]. В нашем исследовании оказалось, что лица в возрасте 50-59 лет наоборот получали кальция с пищей меньше, чем мужчины более старших возрастных групп. Но при изучении потребления кальция в зависимости от места проживания нами были получены те же закономерности, что и по данным Оглоблина Н.А и соавт. [16]: потребление кальция жителями в СЗФО было большим, чем у лиц, проживавших в СФО.

\section{ЗАКЛЮЧЕНИЕ}

Таким образом, наше исследование показало, что у мужчин С $Ф О$, УФО и СФО среди наиболее распространенных клинических ФР ОП и ОП-переломов оказались курение, переломы в анамнезе и различные причины вторичного ОП. 85 \% мужчин в этих регионах не получали с продук- 
тами питания адекватного количества кальция. Более 50\% обследованных лиц имели 2 и более ФР ОП-переломов. Частота ОП составила 21\%. У мужчин с 2-мя и более ФР шанс выявления ОП был в 2,7 раза выше по сравнению с теми, кто ФР ОП-переломов не имел.

\section{ДОПОЛНИТЕЛЬНАЯ ИНФОРМАЦИЯ.}

Конфликт интересов. Авторы декларируют отсутствие явных и потенциальных конфликтов интересов, связанных с публикацией настоящей статьи

\section{СПИСОК ЛИТЕРАТУРЫ | REFERENCES}

1. Bentler SE, Liu L, Obrizan M, et al. The Aftermath of Hip Fracture: Discharge Placement, Functional Status Change, and Mortality. Am J Epidemiol. 2009;170(10):1290-1299. doi: 10.1093/aje/kwp266

2. Hernlund $E$, Svedbom A, Ivergård M, et al. Osteoporosis in the European Union: medical management, epidemiology and economic burden. Archives of Osteoporosis. 2013;8(1-2). doi: 10.1007/s11657-013-0136-1

3. Johnell O, Kanis JA. An estimate of the worldwide prevalence and disability associated with osteoporotic fractures. Osteoporos Int. 2006;17(12):1726-1733. doi: 10.1007/s00198-006-0172-4

4. Беневоленская Л.И. Руководство по остеопорозу. М.: Бином, 2003. [Benevolenskaya LI. Rukovodstvo po osteoporozu. Moscow: Binom; 2003 (In Russ)].

5. Ершова О.Б., Белова К.Ю., Барышева Ю.В. и др. Многофакторность развития остеопороза: обзор научных исследований кафедры терапии Института последипломного образования Ярославского государственного медицинского университета. // Научно-практическая ревматология. - 2016. - Т. 54. - №. 2. С. 187-190. [Ershova OB, Belova KY, Barysheva YV, et al. The Mutifactorial Pattern of Osteoporosis: A Review of the Researches of the Department of Therapy, Institute of Postgraduate Education, Yaroslavl State Medical University. Rheumatology Science and Practice. 2016;54(2):187-190. (In Russ.)] doi: 10.14412/1995-4484-2016-187-190

6. Becker DJ, Kilgore ML, Morrisey MA. The Societal Burden of Osteoporosis. Current Rheumatology Reports. 2010;12(3):186-191. doi: 10.1007/s11926-010-0097-y

7. Khosla S, Amin S, Orwoll E. Osteoporosis in Men. Endocr Rev. 2008;29(4):441-464. doi: 10.1210/er.2008-0002

8. Schuit SCE, van der Klift M, Weel AEAM, et al. Fracture incidence and association with bone mineral density in elderly men and women: the Rotterdam Study. Bone. 2004;34(1):195-202. doi: 10.1016/j.bone.2003.10.001

9. Frost M, Wraae K, Abrahamsen B, et al. Osteoporosis and vertebral fractures in men aged 60-74 years. Age Ageing. 2012;41(2):171-177. doi: 10.1093/ageing/afr170

10. Lewis CE, Ewing SK, Taylor BC, et al. Predictors of Non-Spine Fracture in Elderly Men:The MrOS Study. J Bone Miner Res. 2006;22(2):211-219. doi: 10.1359/jbmr.061017

11. Drake MT, Murad MH, Mauck KF, et al. Risk Factors for Low Bone Mass-Related Fractures in Men: A Systematic Review and Meta-Analysis. J Clin Endocr Metab. 2012;97(6):1861-1870. doi: 10.1210/jc.2011-3058

12. Скрипникова И.А., Гурьев А.В., Шальнова С.А. и др. Распространенность клинических факторов, используемых для оценки риска остеопоротических переломов // Профилактическая медицина. - 2016. - Т. 19. - №5. - С. 32. [Skripnikova IA, Guryev AV, Shalnova SA, et al. The prevalence of clinical factors used for risk assessment of osteoporotic fractures. Profilakticheskaya meditsina. 2016;19(5):32. (In Russ.)] doi: 10.17116/profmed201619532-40

13. Никулина С.Ю., Боева Л.Н., Капустина Е.В. и др. Клинико-анамнестические отличия и характеристика состояния костной ткани у мужчин с остеопеническим синдромом. / Сборник тезисов VII Всероссийского эндокринологического конгресса "Достижения персонализированной медицины сегодня-результат практического здравоохранения завтра"; Москва, 2-5 марта 2016 г. - М.: УП Принт, 2015. - C. 343. [Nikulina SYu, Boeva LN, Kapustina EV, et al. Kliniko-anamnesticheskie otlichiya i kharakteristika sostoyaniya kostnoy tkani u muzhchin s osteopenicheskim sindromom. In: Proceedings of the VII Russian endocrinology congess “Dostizheniya personalizirovannoy meditsiny segodnya-rezul'tat prakticheskogo zdravookhraneniya zavtra"; Moscow, 2-5 March 2016. Moscow: UP Print; 2015. P.343 (In Russ.)]

14. Ершова О.Б., Синицына О.С., Белова К.Ю., и др. Результаты анализа факторов риска и абсолютного риска переломов (FRAX) у мужчин с переломами проксимального отдела бедра // Остеопороз и остеопатии. - 2013. - Т. 16. - №1. - С. 3-6. [Ershova OB, Sinitsyna OS, Belova KY, et al. Results of the analysis of risk factors and absolute risk of fracture (FRAX) in men with fractures of the proximal femur. Osteoporosis and Bone Diseases. 2013;16(1):3-6. (In Russ.)] doi: 10.14341/osteo201313-6

15. Ялочкина Т.О., Белая Ж.Е., Рожинская Л.Я. и др. Распространенность переломов и факторы риска их возникновения у больных сахарным диабетом 2 типа, наблюдаемых в амбулаторном медицинском учреждении г. Москвы. / Сборник тезисов VII Всероссийского диабетологического конгресса «Сахарный диабет в XXI веке - время объединения усилий»; Москва, 24-28 февраля 2015 г. - М.: УП Принт, 2015. - C.334-335. [Yalochkina TO, Belaya ZhE, Rozhinskaya LYa, et al. Rasprostranennost' perelomov i faktory riska ikh vozniknoveniya u bol'nykh sakharnym diabetom 2 tipa, nablyudaemykh v ambulatornom meditsinskom uchrezhdenii g. Moskvy. In: Proceedings of the VII Russian diabetology congress "Diabetes mellitus in XXI century - the time to unite efforts"; Moscow, 24-28 February 2015. Moscow: UP Print; 2015. P.334-335. (In Russ.)]

16. Оглоблин Н.А., Спиричев В.Б., Батурин А.К. О потреблении населением России кальция с пищей // Вопросы питания. 2005. - T. 74. - №. 5. - C. 14-17. [Ogloblin NA, Spirichev V., Baturin AK. O potreblenii naseleniem Rossii kal'tsiya s pishchey. Problems of nutrition. 2005; 74(5):14-17. (In Russ.)]

\section{ИНФОРМАЦИЯ ОБ АВТОРАХ [AUTHORS INFO]}

Никитинская Оксана Анатольевна, К.М.н., С.Н.с. лаборатории остеопороза отдела метаболических заболеваний костей и суставов [Оksana A. Nikitinskaya, MD, PhD, senior researcher]; адрес: 115522 Москва, Каширское шоссе д. 34A; телефон: 8 (499) 614-39-65; ORCID: https://orcid.org/0000-00016759-8367; eLibrary SPIN: 4372-8931; e-mail: nikitinskayaox@yandex.ru

Наталья Владимировна Торопцова, - д.м.Н., руководитель лаборатории остеопороза отдела метаболических заболеваний костей и суставов [Natalya V. Toroptsova, MD, PhD]; ORCID: http://orcid.org/0000-0003-4739-4302; eLibrary SPIN: 5650-2058; e-mail: torop@irramn.ru

\section{ЦИТИРОВАТЬ:}

Никитинская О.А., Торопцова Н.В.Факторы риска остеопороза и остеопоротических переломов у мужчин в возрасте 50 лет и старше. // Остеопороз и остеопатии. - 2017. — T. 20. — №1. - C.7-11. doi: 10.14341/osteo201717-11

\section{TO CITE THIS ARTICLE:}

Nikitinskaya OA, Toroptsova NV. Osteoporosis and osteoporotic fractures risk factors in men aged 50 years and older. Osteoporosis and bone diseases. 2017;20(1):7-11. doi: 10.14341/osteo201717-11 